\title{
Engel sinks of fixed points in finite groups
}

\author{
Cristina Acciarri, Pavel Shumyatsky, and Danilo Silveira
}

\begin{abstract}
For an element $g$ of a group $G$, an Engel sink is a subset $\mathscr{E}(g)$ such that for every $x \in G$ all sufficiently long commutators $[x, g, g, \ldots, g]$ belong to $\mathscr{E}(g)$. Let $q$ be a prime, let $m$ be a positive integer and $A$ an elementary abelian group of order $q^{2}$ acting coprimely on a finite group $G$. We show that if for each nontrivial element $a$ in $A$ and every element $g \in C_{G}(a)$ the cardinality of the smallest Engel sink $\mathscr{E}(g)$ is at most $m$, then the order of $\gamma_{\infty}(G)$ is bounded in terms of $m$ only. Moreover we prove that if for each $a \in A \backslash\{1\}$ and every element $g \in C_{G}(a)$, the smallest Engel sink $\mathscr{E}(g)$ generates a subgroup of rank at most $m$, then the rank of $\gamma_{\infty}(G)$ is bounded in terms of $m$ and $q$ only.
\end{abstract}

\section{Introduction}

A group $G$ is called an Engel group if for every $x, g \in G$ the equation $[x, g, g, \ldots, g]=1$ holds, where $g$ is repeated in the commutator sufficiently many times depending on $x$ and $g$. (Throughout the paper, we use the left-normed simple commutator notation $\left.\left[a_{1}, a_{2}, a_{3}, \ldots, a_{r}\right]=\left[\ldots\left[\left[a_{1}, a_{2}\right], a_{3}\right], \ldots, a_{r}\right].\right)$ The classical theorem of Zorn states that a finite Engel group is nilpotent [22]. In a number of recent papers groups that are 'almost Engel' in the sense of restrictions on so-called Engel sinks were considered [10, 11, 12]. An Engel sink of an element $g \in G$ is a set $\mathscr{E}(g)$ such that for every $x \in G$ all sufficiently long commutators $[x, g, g, \ldots, g]$ belong to $\mathscr{E}(g)$, that is, for

2010 Mathematics Subject Classification. 20D10, 20D45, 20F45.

Key words and phrases. Finite groups, Automorphisms, Centralizers, Engel condition.

This work was supported by the Conselho Nacional de Desenvolvimento Científico e Tecnológico (CNPq), and Fundação de Apoio à Pesquisa do Distrito Federal (FAPDF), Brazil. 
every $x \in G$ there is a positive integer $n(x, g)$ such that

$$
[x, \underbrace{g, g, \ldots, g}_{n}] \in \mathscr{E}(g) \quad \text { for all } n \geq n(x, g) .
$$

Engel groups are precisely the groups for which we can choose $\mathscr{E}(g)=$ $\{1\}$ for all $g \in G$. In [11] finite, profinite, and compact groups in which every element has a finite Engel sink were considered. It was shown that compact groups with this property are finite-by-(locally nilpotent). Results for finite groups have to be of quantitative nature. Obviously, in a finite group every element has the smallest Engel sink, so from now on we use the term Engel sink for the minimal Engel sink of $g$, denoted by $\mathscr{E}(g)$, thus eliminating ambiguity in this notation. One of the results obtained in [11] says that if $G$ is a finite group and there is a positive integer $m$ such that $|\mathscr{E}(g)| \leq m$ for all $g \in G$, then $G$ has a normal subgroup of order bounded in terms of $m$ with nilpotent quotient. A subsequent paper [12] dealt with finite groups in which there is a bound on the ranks of the subgroups generated by the Engel sinks. Here, the rank of a finite group is the minimum number $r$ such that every subgroup can be generated by $r$ elements. Let $\gamma_{\infty}(G)=\cap_{i=1}^{\infty} \gamma_{i}(G)$ be the intersection of all terms of the lower central series of a group $G$ (the nilpotent residual of $G$ ). The following results were obtained in [11, Theorem 3.1] and [12, Theorem 1.1], respectively.

Theorem 1.1. Let $G$ be a finite group and $m$ a positive integer.

(1) If $\mathscr{E}(g)$ has at most $m$ elements for every $g \in G$, then the order of $\gamma_{\infty}(G)$ is bounded in terms of $m$ only.

(2) If $\langle\mathscr{E}(g)\rangle$ has rank at most $m$ for every $g \in G$, then the rank of $\gamma_{\infty}(G)$ is bounded in terms of $m$ only.

As usual, $\langle X\rangle$ denotes the subgroup generated by a subset $X$ of $G$. In the present article we consider finite groups $G$ admitting a coprime action by an elementary abelian $q$-group $A$ with certain conditions on Engel sinks of elements of $C_{G}(a)$ for $a \in A^{\#}$. Here $q$ is a prime, $C_{G}(a)$ denotes the subgroup of $G$ formed by the fixed points of the automorphism $a$, while the symbol $A^{\#}$ stands for the set of nontrivial elements of $A$. Recall that an action of $A$ on $G$ is called coprime if $(|A|,|G|)=1$. Our purpose is to establish the following theorems.

Theorem 1.2. Let $m$ be a positive integer, $q$ a prime, and $A$ an elementary abelian group of order $q^{2}$ acting coprimely on a finite group $G$. If $\mathscr{E}(g)$ has at most $m$ elements for every $g \in C_{G}(a)$ and every $a \in A^{\#}$, then the order of $\gamma_{\infty}(G)$ is bounded in terms of $m$ only. 
Theorem 1.3. Let $m$ be a positive integer, $q$ a prime, and $A$ an elementary abelian group of order $q^{2}$ acting coprimely on a finite group $G$. If $\langle\mathscr{E}(g)\rangle$ has rank at most $m$ for every $g \in C_{G}(a)$ and every $a \in A^{\#}$, then the rank of $\gamma_{\infty}(G)$ is bounded in terms of $m$ and $q$ only.

The surprising aspect of Theorem 1.2 is that the order of $\gamma_{\infty}(G)$ turns out to be independent of the order of $A$. We do not know if a similar phenomenon also holds under the hypotheses of Theorem 1.3 . Quite possibly, Theorem 1.3 can be strengthened by showing that the rank of $\gamma_{\infty}(G)$ is bounded in terms of $m$ only. This seems an interesting question for future projects.

Formally, the proofs of Theorems 1.2 and 1.3 follow similar plans but in fact there are significant differences. In particular, the proof of Theorem 1.2 uses the observation that if a finite group $G$ admits a coprime action by an elementary abelian $q$-group $A$ such that $C_{G}(a)$ has order at most $m$ for each $a \in A^{\#}$, then the order of $G$ is bounded in terms of $m$ only and is independent of the order of $A$ (see Lemma 3.1 in Section 3). We think that there is no analog of that observation for the case where $C_{G}(a)$ has rank at most $m$ for each $a \in A^{\#}$. This partially explains why in Theorem 1.3 the rank of $\gamma_{\infty}(G)$ is bounded in terms of both $m$ and $q$.

Note that both parts of Theorem 1.1 can be obtained as particular cases of Theorems 1.2 and 1.3 , respectively, where the action of $A$ on $G$ is trivial. On the other hand, Theorem 1.1 is used in the proof of both Theorems 1.2 and 1.3 .

In the next section we include some (mostly well-known) auxiliary lemmas. The proof of Theorem 1.2 is given in Section 3. The proof of Theorem 1.3 is given in Section 4 .

\section{Preliminaries}

Throughout the paper we use, without special references, the following well-known properties of coprime actions (see for example [3. 5.3.6, 6.2,2, 6.2.4] and [14, Theorem 8.2.6]). Let $A$ be a group acting coprimely on a finite group $G$. We will use the usual notation for commutators $[g, a]=g^{-1} g^{a}$ and $[G, A]=\langle[g, a] \mid g \in G, a \in A\rangle$. Then:

- $[G, A]=[G, A, A]$;

- If $N$ is an $A$-invariant normal subgroup of $G$, then $C_{G / N}(A)=$ $C_{G}(A) N / N$;

- If $A$ is a noncyclic abelian group, then $G$ is generated by the subgroups $C_{G}(B)$, where $A / B$ is cyclic;

- $G$ has an $A$-invariant Sylow $p$-subgroup for each prime $p$ dividing $|G|$. 
If $G$ is soluble, then there exist $A$-invariant Hall $\pi$-subgroups of $G$, for any nonempty subset $\pi$ of prime divisors of $|G|$.

In what follows, let $\mathbf{r}(G)$ denote the rank of a finite group $G$, and we use the expression " $\{a, b, \ldots\}$-bounded" to abbreviate the expression "bounded from above by a function that depends on $a, b, \ldots$ only".

We will also require the following well-known facts.

Lemma 2.1. Let $N, H_{1}, \ldots, H_{t}$ be subgroups of a group $G$ with $N$ being normal. If $K=\left\langle H_{1}, \ldots, H_{t}\right\rangle$, then $[N, K]=\left[N, H_{1}\right] \cdots\left[N, H_{t}\right]$.

For the proof of the next lemma see, for example, [12, Lemma 2.6].

Lemma 2.2. Suppose that a group $A$ acts by automorphisms on a group $G$. If $A=\left\langle a_{1}, \ldots, a_{t}\right\rangle$, then $[G, A]=\left[G, a_{1}\right] \cdots\left[G, a_{t}\right]$.

The next results relate Engel sinks in a finite group to coprime actions. They were established in [11, Lemma 3.2] and [12, Lemma 2.7 , respectively.

Lemma 2.3. Let $P$ be a finite $p$-subgroup of a group $G$, and $g \in G$ a $p^{\prime}$-element normalizing $P$. Then

(1) the order of $[P, g]$ is bounded in terms of the cardinality of the Engel sink $\mathscr{E}(g)$;

(2) the subgroup $[P, g]$ is contained in $\langle\mathscr{E}(g)\rangle$.

In the next lemma we collect two useful results.

Lemma 2.4. Let $p$ be a prime, $P$ a finite $p$-group, and $A$ a $p^{\prime}$-group of automorphisms of $P$.

(1) If $|[P, a]| \leq m$ for every $a \in A$, then $|A|$ and $|[P, A]|$ are $m$ bounded.

(2) If $\mathbf{r}([P, a]) \leq m$ for every $a \in A$, then $\mathbf{r}(A)$ and $\mathbf{r}([P, A])$ are m-bounded.

Proof. The proof of Part (2) can be found in [12, Proposition 3.1]. Let us prove Part (1).

Without loss of generality we can assume that $P=[P, A]$. It is sufficient to bound the order of $A$ since a bound on the order of $P$ will follow simply observing, by Lemma 2.2 , that $[P, A]=\prod_{a \in A}[P, a]$. Thus, let us show that the order of $A$ is $m$-bounded.

Let $B$ be the group of automorphisms that $A$ induces on the quotient $P / \Phi(P)$. By [10, Lemma 2.3] the order of $B$ is $m$-bounded. Suppose that $B$ has order smaller than $A$. This means that $A$ contains a nontrivial element $a$ which acts trivially on $P / \Phi(P)$. This in turn means that $P=C_{P}(a) \Phi(P)$. Since the Frattini subgroup $\Phi(P)$ is a 
non-generating set, we conclude that $P=C_{P}(a)$ and so $a=1$. Thus, $|A|=|B|$ and we are done.

The following result was obtained by Kovács [6] for soluble groups, and extended, to the general case, independently by Guralnick [4] and Lucchini [16] using the classification of finite simple groups.

Lemma 2.5. If $d$ is the maximum of the ranks of the Sylow subgroups of a finite group, then the rank of this group is at most $d+1$.

Let $F(G)$ denote the Fitting subgroup of a group $G$. Write $F_{0}(G)=$ $1, F_{1}(G)=F(G)$ and let $F_{i+1}(G)$ be the inverse image of $F\left(G / F_{i}(G)\right)$. If $G$ is soluble, then the least number $h$ such that $F_{h}(G)=G$ is called the Fitting height $h(G)$ of $G$. The next result is well-known (see for example [2, Lemma 2.4] for the proof).

Lemma 2.6. If $G$ is a finite group of Fitting height 2, then $\gamma_{\infty}(G)=$ $\prod_{p}\left[F_{p}, H_{p^{\prime}}\right]$, where $F_{p}$ is a Sylow p-subgroup of $F(G)$ and $H_{p^{\prime}}$ is a Hall $p^{\prime}$-subgroup of $G$.

\section{Proof of Theorem 1.2}

In this section we present a proof of Theorem 1.2 . Let us start with the following lemma.

Lemma 3.1. Let $m$ be a positive integer and $q$ a prime. Let $A$ be an elementary abelian group of order $q^{2}$ acting coprimely on a finite group $G$ in such a manner that $\left|C_{G}(a)\right| \leq m$ for every $a \in A^{\#}$. Then the order of $G$ is m-bounded.

Proof. Note that $A$ normalizes some Sylow $p_{i}$-subgroup $P_{i}$ of $G$, for each prime divisor $p_{i}$ of $|G|$. Since $|G|=\left|P_{1}\right| \cdots\left|P_{s}\right|$, it is enough to show that $\left|P_{i}\right|$ is $m$-bounded for every $p_{i}$. Indeed, let $C$ be the maximum possible value for $\left|P_{i}\right|$, which is an $m$-bounded number. Then every $p_{i} \leq C$ and, so $|G|$ is $m$-bounded as well.

Thus, without loss of generality, we can assume that $G$ is a $p$-group for some $p \neq q$. Then $G=\prod_{a \in A^{\#}} C_{G}(a)$ and so $G$ has order at most $m^{q+1}$, since $A$ has exactly $q+1$ cyclic subgroups.

If $q \leq m$ !, then $|G| \leq m^{m !+1}$, and we are done. Assume that $q>m$ !. Let $a \in A^{\#}$. Note that the centralizer $C_{G}(a)$ is $A$-invariant since $A$ is abelian. Observe that the order of any automorphism of $C_{G}(a)$ is at most $(m-1)$ ! and therefore $A$ induces the trivial action on $C_{G}(a)$. In other words $C_{G}(a) \leq C_{G}(A)$. This implies that all centralizers are equal and, in particular, that $G=C_{G}(a)$ for any $a \in A^{\#}$. Hence $|G| \leq m$, as desired. 
The first step in the proof of Theorem 1.2 deals with the case where $G$ is soluble. We require the following result due to Thompson [20] (see also [21, Corollary 3.2]).

Theorem 3.2. Let $A$ be a soluble coprime group of automorphisms of a finite soluble group $G$. Then the Fitting height of $G$ is bounded in terms of $h\left(C_{G}(A)\right)$ and the number of prime factors of $|A|$ counting multiplicities.

Lemma 3.3. Theorem 1.2 holds if $G$ is a soluble group.

Proof. Suppose that $G$ is soluble. It follows from Theorem 1.1(1) that $\gamma_{\infty}\left(C_{G}(a)\right)$ has $m$-bounded order, for each $a$ in $A^{\#}$. Therefore $h\left(C_{G}(a)\right)$ is $m$-bounded for any $a$ in $A^{\#}$ and, by Theorem $3.2, h(G)$ is $m$-bounded as well. The lemma now can be proved by induction on $h(G)$.

If $h(G)=1$, then the result is obvious. Suppose that $h(G)=2$. By Lemma 2.6, we have $\gamma_{\infty}(G)=\prod_{p}\left[F_{p}, H_{p^{\prime}}\right]$, where $F_{p}$ is a Sylow $p$-subgroup of $F(G)$, while $H_{p^{\prime}}$ is a Hall $p^{\prime}$-subgroup of $G$ that can be chosen $A$-invariant and the product is taken over all prime divisors of $|F(G)|$.

First observe that $\left|\left[F_{p}, H_{p^{\prime}}\right]\right|=\left|\left[F_{p}, \bar{H}_{p^{\prime}}\right]\right|$, where $\bar{H}_{p^{\prime}}=H_{p^{\prime}} / C_{H_{p^{\prime}}}\left(F_{p}\right)$. By Lemma 2.3(1), the order of $\left[F_{p}, h\right]$ is $m$-bounded, for any $h \in$ $C_{\bar{H}_{p^{\prime}}}(a)$. It follows from Lemma 2.4(1) that the order of $\left[F_{p}, C_{\bar{H}_{p^{\prime}}}(a)\right]$ is $m$-bounded for any $a \in A^{\#}$. Since $C_{\left[F_{p}, \bar{H}_{p^{\prime}}\right]}(a) \leq\left[F_{p}, C_{\bar{H}_{p^{\prime}}}(a)\right]$ for any $a \in A^{\#}$, in view of Lemma 3.1 , we conclude that $\left|\left[F_{p}, \bar{H}_{p^{\prime}}\right]\right|$ is $m$-bounded.

Let $C$ be the maximum possible value for $\left|\left[F_{p}, H_{p^{\prime}}\right]\right|$, which is an $m$ bounded number. Suppose that $p>C$. Since $F_{p}$ cannot have nontrivial subgroups of order at most $C$, deduce that $\left[F_{p}, H_{p^{\prime}}\right]=1$. Let $p_{1}, \ldots, p_{s}$ be the primes dividing $\left|\gamma_{\infty}(G)\right|$. It follows from the observation above that each $p_{i}$ is at most $C$. Therefore $\left|\gamma_{\infty}(G)\right| \leq C^{s}$, which is an $m$ bounded number, as claimed.

Finally, suppose that $h(G)>2$ and let $N=F_{2}(G)$ be the second term of the Fitting series of $G$. It is clear that the Fitting height of $G / \gamma_{\infty}(N)$ is $h(G)-1$ and $\gamma_{\infty}(N) \leq \gamma_{\infty}(G)$. The above argument shows that $\left|\gamma_{\infty}(N)\right|$ is $m$-bounded. Hence, by induction the order of $\gamma_{\infty}(G) / \gamma_{\infty}(N)$ is $m$-bounded. The result follows.

In order to deal with the case when $G$ is a nonsoluble group, first we consider the special case where $G$ is a direct product of nonabelian simple groups. 
A proof of the following theorem can be found, for example, in [1, Corollary 3.5].

Theorem 3.4. Let $q$ be a prime and $A$ an elementary abelian group of order $q^{2}$ acting on a finite $q^{\prime}$-group $G$ in such a way that $C_{G}(a)$ is nilpotent for every nontrivial $a \in A$. Then $G$ is soluble.

In the next lemma we will also use the fact that if $A$ is any coprime group of automorphisms of a finite simple group, then $A$ is cyclic (see, for example, [5. Lemma 2.7]).

Let $M_{1}=M_{1}(m)$ be the maximal possible value of $\left|\gamma_{\infty}(G)\right|$ in Theorem 1.1(1) and let $M_{2}=M_{2}(m)$ be the maximal possible value of $\left|\gamma_{\infty}(G)\right|$ in Lemma 3.3. Set $M=\max \left\{M_{1}, M_{2}\right\}$.

Lemma 3.5. Let $G$ and $A$ be as in Theorem 1.2. Moreover assume that $G=S_{1} \times \cdots \times S_{t}$ is a direct product of $t$ nonabelian simple groups $S_{i}$. Then the order of $G$ is m-bounded.

ProOF. First, we prove that each direct factor $S_{i}$ has order at most $M$. If $S_{i}$ is $A$-invariant, then $S_{i}$ is contained in $C_{G}(a)$ for some $a \in A^{\#}$ and so $S_{i} \leq \gamma_{\infty}\left(C_{G}(a)\right)$. Hence $\left|S_{i}\right| \leq M$, since by Theorem $1.1(1)$ and the above observation $\left|\gamma_{\infty}\left(C_{G}(a)\right)\right| \leq M$. Suppose now that $S_{i}$ is not $A$-invariant. Choose $a \in A^{\#}$ such that $S_{i}^{a} \neq S_{i}$. Write $T=S_{i} \times S_{i}^{a} \times \cdots \times S_{i}^{a^{q-1}}$. Since $S_{i}^{a} \neq S_{i}$, it is easy to see that $C_{T}(a)$ is exactly the "diagonal" subgroup of $T$, that is,

$$
C_{T}(a)=\left\{\left(g, g^{a}, \ldots, g^{a^{q-1}}\right) \mid g \in S_{i}\right\} .
$$

Thus, $C_{T}(a)$ is isomorphic to $S_{i}$ and Theorem $1.1(1)$ implies that $\left|S_{i}\right| \leq$ $M$, as claimed.

Next, we show that if $q \geq M$ !, then $A$ acts trivially on $G$. Suppose that this is false and $q \geq M$ ! while $A$ acts nontrivially on $G$.

Let $K$ be a minimal normal $A$-invariant subgroup of $G$. Thus, $K$ is direct product of subgroups $S^{a}$, where $S$ is one of $S_{i}$ and $a$ ranges through $A$. As shown above, if $K=S$, then $|K| \leq M$. Since for any $a \in A^{\#}$ the order of $a$ is bigger that $M$ !, it follows that $K \leq C_{G}(A)$, a contradiction. Suppose now that $K \neq S$. In this case either $K$ is a product of $q$ simple factors or a product of $q^{2}$ ones. In the former case we have $K=S \times S^{a} \cdots \times S^{a^{q-1}}$ for some $a \in A$ and there exists $b \in A$ such that $S^{b}=S$. If $b$ centralizes $S$, then $K \leq C_{G}(b)$ and so $|K| \leq M$ whence again we deduce that $A$ centralizes $K$ since $q \geq M$ !. This is a contradiction because $S \neq S^{a}$. Hence, $b$ does not centralize $S$. Note that $C_{K}(a) \cong S$ is the "diagonal" subgroup of $K$. Observe that $C_{K}(a)$ is $A$-invariant and the order of $C_{K}(a)$ is at most $M$. Taking 
into account that $q \geq M$ ! we deduce that $A$ acts trivially on $C_{K}(a)$. In particular, $C_{K}(a) \leq C_{K}(b)$. On the other hand,

$$
C_{K}(b)=C_{S}(b) \times C_{S}(b)^{a} \times \ldots C_{S}(b)^{a^{q-1}}
$$

and so $C_{K}(a)$ is not contained in $C_{K}(b)$.

Therefore we can assume that $K$ is a product of $q^{2}$ simple factors which are transitively permuted by $A$. Recall that minimal nonnilpotent finite groups are soluble [19, Theorem 9.1.9]. Therefore $S$ contains a soluble subgroup $D$ which is not nilpotent. Thus $\left|\gamma_{\infty}(D)\right| \geq 3$. Then $D_{0}=\left\langle D^{A}\right\rangle$ is a soluble $A$-invariant subgroup of $K$ such that

$$
\left|\gamma_{\infty}\left(D_{0}\right)\right| \geq 3^{q^{2}}
$$

This is a contradiction since $q \geq M$ while $\left|\gamma_{\infty}\left(D_{0}\right)\right| \leq M$.

This proves that either $A$ acts trivially on $G$ or $q \leq M$ !. In the former case $|G| \leq M$ and we are done. Thus, assume that $q \leq M$ !.

Write $G=K_{1} \times \cdots \times K_{s}$ where each $K_{i}$ is a minimal normal $A$ invariant subgroup of $G$. Since, as proved above, each factor $S_{i}$ has order at most $M$ and $q$ is now $M$-bounded, each $K_{i}$ is of $m$-bounded order. Therefore, it is sufficient to bound $s$.

By Theorem 3.4, for every $i$ there exists $a \in A^{\#}$ such that $C_{K_{i}}(a)$ is not nilpotent. Therefore, $\gamma_{\infty}\left(C_{K_{i}}(a)\right) \neq 1$. Since $\gamma_{\infty}\left(C_{G}(a)\right)$ has order at most $M$, it follows that $\gamma_{\infty}\left(C_{K_{i}}(a)\right)$ can be nontrivial for at most $M$ indexes $i$. Taking into account that there are only $q+1$ nontrivial proper subgroups in $A$, we conclude that $s \leq(q+1) M \leq(M !+1) M$.

Every finite group $G$ has a normal series each of whose factors either is soluble or is a nonempty direct product of nonabelian simple groups. The nonsoluble length $\lambda(G)$ of a finite group $G$ is defined as the minimum number of nonsoluble factors in a normal series of that kind.

We will require the following two results.

Theorem 3.6 ([8, Theorem 1.3]). Let $A$ be a coprime group of automorphisms of a finite group $G$. Then the nonsoluble length of $G$ is bounded in terms of $\lambda\left(C_{G}(A)\right)$ and the number of prime factors of $|A|$ counting multiplicities.

Lemma 3.7 ([17, Lemma 3.2]). Let $K$ be a nonabelian finite simple group and $p$ a prime. There exists a prime $s$, different from $p$, such that $K$ is generated by two Sylow s-subgroups.

We are now ready to prove Theorem 1.2 in the general case.

Proof of Theorem 1.2. In view of Lemma 3.3 we can assume that $G$ is not soluble. By Theorem 1.1(1), the order of $\gamma_{\infty}\left(C_{G}(a)\right)$ is 
$m$-bounded for any $a \in A^{\#}$. Therefore $\lambda\left(\gamma_{\infty}\left(C_{G}(a)\right)\right)$ is $m$-bounded, and so $\lambda\left(C_{G}(a)\right)$ is $m$-bounded as well. Thus Theorem 3.6 guarantees that $\lambda(G)$ is $m$-bounded, and we can use induction on $\lambda(G)$.

First, assume that $\lambda(G)=1$ and $G=G^{\prime}$. Let $R(G)$ be the soluble radical of $G$. It follows that $G / R(G)$ is a product of nonabelian simple groups. Since $R(G)$ is an $A$-invariant soluble group, Lemma 3.3 ensures that the order of $\gamma_{\infty}(R(G))$ is $m$-bounded. Note that if the nilpotent residual of $G / \gamma_{\infty}(R(G))$ has $m$-bounded order, then $\gamma_{\infty}(G)$ has $m$ bounded order too. Thus, without loss of generality, we assume that $\gamma_{\infty}(R(G))=1$ and $R(G)=F(G)$.

Next we will show that the order of $[F(G), G]$ is $m$-bounded. Since $F(G)$ is nilpotent we have

$$
[F(G), G]=\left[P_{1}, G\right] \cdots\left[P_{l}, G\right]
$$

where the subgroups $P_{i}$ are Sylow $p_{i}$-subgroups of $F(G)$. It is sufficient to show that the order of $[P, G]$ is $m$-bounded for each $p$-Sylow subgroup $P$ of $F(G)$. Note that, considering the quotient of $G$ by the Hall $p^{\prime}$ subgroup of $F(G)$ and taking into account that the soluble radical of $G$ is nilpotent, we can assume that $F(G)=P$ is a $p$-group.

Since $\lambda(G)=1$ and $G=G^{\prime}$, the quotient $G / F(G)$ is a direct product of nonabelian simple groups. By Lemma $3.5 G / P$ has $m$ bounded order. Write $G=G_{1} G_{2} \ldots G_{t}$, where $G_{i} / P$ is a minimal normal $A$-invariant subgroup of $G / P$. Obviously $[P, G]=\prod_{i}\left[P, G_{i}\right]$. Thus it is sufficient to show that the order of $\left[P, G_{i}\right]$ is $m$-bounded. Hence, without loss of generality, we can assume that $G=G_{1}$, that is, $G / P$ is a product of isomorphic nonabelian simple groups transitively permuted by $A$. By Lemma 3.7 the quotient $G / P$ is generated by the image of two Sylow $s$-subgroup $H_{1}$ and $H_{2}$, where $s$ is a prime different from $p$. Furthermore, $H_{1}$ and $H_{2}$ are conjugate of an $A$-invariant Sylow $s$-subgroup $K$.

Note that, by Lemma 3.3 , the order of $\gamma_{\infty}(P K)$ is $m$-bounded because $P K$ is $A$-invariant and soluble. We also have $[P, K]=[P, K, K]$ and consequently $[P, K] \leq \gamma_{\infty}(P K)$. Since $H_{1}$ and $H_{2}$ are conjugate to $K$, it follows that both $\left[P, H_{1}\right]$ and $\left[P, H_{2}\right]$ have $m$-bounded order.

Let $H=\left\langle H_{1}, H_{2}\right\rangle$. Thus $G=P H$. Since $G=G^{\prime}$ we deduce that $G=[P, H] H$ because $[P, H] H$ is normal and the quotient $G /[P, H] H$ is a $p$-group. Furthermore, $[P, G]=[P, H]$. Indeed, we have

$$
[P, G]=[P,[P, H] H]=[P,[P, H]][P, H] .
$$

Since $[P, H]$ is normal in $P$ and $G=P H$, the subgroup $[P, H]$ is also normal in $G$ and so $[[P, H], P] \leq[P, H]$. 
Observe that $[P, H]=\left[P, H_{1}\right]\left[P, H_{2}\right]$. Thus the order of $[P, H]$ is $m$-bounded. Passing to the quotient $G /[P, G]$ we can assume that $P=Z(G)$. In view of Lemma 3.5, we know that $G / Z(G)$ has $m$ bounded order. By quantitative version of Schur's Theorem (see, for example, [18, p. 102]) the order of $G^{\prime}=G$ is $m$-bounded as well.

Now we deal with the case where $\lambda(G)=1$ and $G \neq G^{\prime}$. Let $G^{(d)}$ be the last term of the derived series of $G$. The argument in the previous paragraph shows that $G^{(d)}$ has $m$-bounded order. Hence, the order of $\gamma_{\infty}(G)$ is $m$-bounded since $G / G^{(d)}$ is soluble. This proves the theorem in the particular case where $\lambda(G) \leq 1$.

Assume that $\lambda(G) \geq 2$. Let $T$ be a characteristic subgroup of $G$ such that $\lambda(T)=\lambda(G)-1$ and $\lambda(G / T)=1$. By induction, the order of $\gamma_{\infty}(T)$ is $m$-bounded. Since $\lambda\left(G / \gamma_{\infty}(T)\right)=1$, the order of the nilpotent residual of $G / \gamma_{\infty}(T)$ is $m$-bounded too. Consequently, we conclude that $\gamma_{\infty}(G)$ has $m$-bounded order. This completes the proof.

\section{Proof of Theorem 1.3}

In this section we prove Theorem 1.3. Similarly to what was done in the previous section, the first step in the proof deals with the case of soluble groups.

Lemma 4.1. Theorem 1.3 holds if $G$ is a soluble group.

Proof. Assume that $G$ is soluble. By Theorem 1.1(2) the rank of $\gamma_{\infty}\left(C_{G}(a)\right)$ is $m$-bounded for each $a \in A^{\#}$. In [7, Lemma 8] it was proved that a finite soluble group of rank $r$ has $r$-bounded Fitting height. Hence we deduce that $h\left(\gamma_{\infty}\left(C_{G}(a)\right)\right)$ is $m$-bounded and so, in particular, $C_{G}(a)$ has $m$-bounded Fitting height for any $a \in A^{\#}$. Now Theorem 3.2 implies that $h(G)$ is $m$-bounded. The lemma now can be proved by induction on $h(G)$. If $h(G)=1$, then the result is obvious.

Next, we are going to establish that if $h(G)=2$, then the rank of $\gamma_{\infty}(G)$ is $\{m, q\}$-bounded. By Lemma 2.6, we have $\gamma_{\infty}(G)=\prod_{p}\left[F_{p}, H_{p^{\prime}}\right]$, where $F_{p}$ is a Sylow $p$-subgroup of $F(G)$, while $H_{p^{\prime}}$ is a Hall $p^{\prime}$-subgroup of $G$ that can be chosen $A$-invariant and the product is taken over all prime divisors of $|F(G)|$. It follows from Lemma $2.3(2)$ that $\left[F_{p}, h\right]$ has $m$-bounded rank for any $h \in C_{H_{p^{\prime}}}(a)$. Hence, by Lemma 2.4(2), we deduce that $\mathbf{r}\left(\left[F_{p}, C_{H_{p^{\prime}}}(a)\right]\right)$ is $m$-bounded for each $a \in A^{\#}$. Since $H_{p^{\prime}}=\left\langle C_{H_{p^{\prime}}}(a) \mid a \in A^{\#}\right\rangle$, Lemma 2.1 tells us that

$$
\left[F_{p}, H_{p^{\prime}}\right]=\prod_{a \in A^{\#}}\left[F_{p}, C_{H_{p^{\prime}}}(a)\right]
$$


Therefore the rank of $\left[F_{p}, H_{p^{\prime}}\right]$ is $\{m, q\}$-bounded. By Lemma 2.5 we conclude that $\gamma_{\infty}(G)$ has $\{m, q\}$-bounded rank, as well.

Finally, suppose that $h(G)>2$ and let $N=F_{2}(G)$. By induction the rank of $\gamma_{\infty}(G) / \gamma_{\infty}(N)$ is $\{m, q\}$-bounded. The result follows since $\mathbf{r}\left(\gamma_{\infty}(G)\right) \leq \mathbf{r}\left(\gamma_{\infty}(G) / \gamma_{\infty}(N)\right)+\mathbf{r}\left(\gamma_{\infty}(N)\right)$.

Now we consider the special case where $G$ is a direct product of nonabelian simple groups. Most of the argument follows the scheme as in the proof of Lemma 3.5

Lemma 4.2. Let $G$ and $A$ be as in Theorem 1.3. Assume also that $G=S_{1} \times \cdots \times S_{t}$ is a direct product of $t$ nonabelian simple groups $S_{i}$. Then $t$ is $\{m, q\}$-bounded and the rank of each $S_{i}$ is $m$-bounded.

Proof. First, we prove that each direct factor $S_{i}$ has $m$-bounded rank. If $S_{i}$ is $A$-invariant, then $S_{i}$ is contained in $C_{G}(a)$ for some $a \in A^{\#}$ and so $S_{i} \leq \gamma_{\infty}\left(C_{G}(a)\right)$. Hence $S_{i}$ has $m$-bounded rank by Theorem 1.1(2). Suppose now that $S_{i}$ is not $A$-invariant. Choose $a \in A^{\#}$ such that $S_{i}^{a} \neq S_{i}$. Write $S=S_{i} \times S_{i}^{a} \times \cdots \times S_{i}^{a^{q-1}}$. Since $S_{i}^{a} \neq S_{i}$ it is easy to see that $C_{S}(a)$ is exactly the "diagonal" subgroup of $S$, that is,

$$
C_{S}(a)=\left\{\left(g, g^{a}, \ldots, g^{a^{q-1}}\right) \mid g \in S_{i}\right\} .
$$

Thus, $C_{S}(a)$ is isomorphic to $S_{i}$ and Theorem 1.1(2) implies that $S_{i}$ has $m$-bounded rank, as claimed.

Now, we will show that $t$ is an $\{m, q\}$-bounded number. Write $G=K_{1} \times \cdots \times K_{s}$ where each $K_{i}$ is a minimal normal $A$-invariant subgroup of $G$. Then each $K_{i}$ is a product of at most $|A|$ simple factors and so $t \leq|A| s$. Therefore, it is sufficient to bound $s$.

Let $S_{j}$ be a direct factor of $K_{i}$. Let us show that for every $i$ there exists $a \in A^{\#}$ such that $C_{K_{i}}(a)$ contains a subgroup isomorphic to some $S_{j}$. If $S_{j}$ is $A$-invariant, then $S_{j}$ is contained in $C_{K_{i}}(a)$ for some $a \in A^{\#}$. Suppose now that $S_{j}$ is not $A$-invariant. Choose $b \in A^{\#}$ such that $S_{j}^{b} \neq S_{j}$. As shown above, the centralizer $C_{S}(b)$ is exactly the "diagonal" subgroup of $S=S_{j} \times S_{j}^{b} \times \cdots \times S_{j}^{b^{q-1}}$. Hence $C_{S}(b)$ is isomorphic to $S_{j}$ and it is contained in $C_{K_{i}}(b)$, as claimed.

Therefore, for every $i$, there exists $a \in A^{\#}$ such that $\gamma_{\infty}\left(C_{K_{i}}(a)\right)$ has even order, so in particular $\gamma_{\infty}\left(C_{K_{i}}(a)\right) \neq 1$. Note that $\mathbf{r}\left(\gamma_{\infty}\left(C_{G}(a)\right)\right)$ is $m$-bounded by Theorem 1.1(2), for any $a \in A^{\#}$. Let $r$ be the maximal value of $\mathbf{r}\left(\gamma_{\infty}\left(C_{G}(a)\right)\right)$ when $a$ ranges through $A$, that is an $\{m, q\}$ bounded number. Since $\gamma_{\infty}\left(C_{G}(a)\right)=\gamma_{\infty}\left(C_{K_{1}}(a)\right) \times \cdots \times \gamma_{\infty}\left(C_{K_{s}}(a)\right)$, it follows that $s \leq r$, so it is $m$-bounded as desired.

We are now ready to prove Theorem 1.3 in the general case. 
Proof of TheOrem 1.3. In view of Lemma 4.1 we can assume that $G$ is not soluble. By Theorem 1.1 (2), the rank of $\gamma_{\infty}\left(C_{G}(a)\right)$ is $m$-bounded for any nontrivial element $a$ in $A$. Now [7, Lemma 8] tells us that each soluble subgroup of $\gamma_{\infty}\left(C_{G}(a)\right)$ has $m$-bounded Fitting height. It was established in [9, Corollary 1.2] that the nonsoluble length of a finite group does not exceed the maximum Fitting height of its soluble subgroups. Therefore $\lambda\left(\gamma_{\infty}\left(C_{G}(a)\right)\right)$ is $m$-bounded, and so $\lambda\left(C_{G}(a)\right)$ is $m$-bounded as well. Thus Theorem 3.6 guarantees that $\lambda(G)$ is $m$-bounded, and we can use induction on $\lambda(G)$.

First, assume that $\lambda(G)=1$ and $G=G^{\prime}$. Let $R(G)$ be the soluble radical of $G$. It follows that $G / R(G)$ is a product of nonabelian simple groups. Since $R(G)$ is an $A$-invariant soluble group, Lemma 4.1 ensures that $\mathbf{r}\left(\gamma_{\infty}(R(G))\right)$ is $\{m, q\}$-bounded. Note that if the nilpotent residual of $G / \gamma_{\infty}(R(G))$ has $\{m, q\}$-bounded rank, then $\gamma_{\infty}(G)$ has $\{m, q\}$-bounded rank too. Thus, without loss of generality, we assume that $\gamma_{\infty}(R(G))=1$ and $R(G)=F(G)$.

Next we will show that the rank of $[F(G), G]$ is $\{m, q\}$-bounded. Since $F(G)$ is nilpotent we have

$$
[F(G), G]=\left[P_{1}, G\right] \cdots\left[P_{l}, G\right]
$$

where the subgroups $P_{i}$ are Sylow $p_{i}$-subgroups of $F(G)$. In view of Lemma 2.5 it is enough to show that the rank of $[P, G]$ is $\{m, q\}$ bounded for each Sylow $p$-subgroup $P$ of $F(G)$. Note that, considering the quotient of $G$ by the Hall $p^{\prime}$-subgroup of $F(G)$ and taking into account that the soluble radical of $G$ is nilpotent, we can assume that $F(G)=P$ is a $p$-group.

Since $\lambda(G)=1$ and $G=G^{\prime}$, the quotient $G / P$ is a direct product of nonabelian simple groups. By Lemma $4.2 G / P$ has $\{m, q\}$ boundedly many simple factors. Write $G=G_{1} G_{2} \ldots G_{t}$, where $t$ is $\{m, q\}$-bounded and $G_{i} / P$ is a minimal normal $A$-invariant subgroup of $G / P$. Obviously $[P, G]=\prod_{i}\left[P, G_{i}\right]$. Thus it is sufficient to show that the rank of $\left[P, G_{i}\right]$ is $\{m, q\}$-bounded. Hence, without loss of generality, we can assume that $G=G_{1}$, that is, $G / P$ is a product of isomorphic nonabelian simple groups transitively permuted by $A$. By Lemma 3.7 the quotient $G / P$ is generated by the image of two Sylow $s$-subgroup $H_{1}$ and $H_{2}$, where $s$ is a prime different from $p$. Furthermore, $H_{1}$ and $H_{2}$ are conjugate of an $A$-invariant Sylow $s$-subgroup $K$.

Observe that, by Lemma 4.1, $\mathbf{r}\left(\gamma_{\infty}(P K)\right)$ is $\{m, q\}$-bounded because $P K$ is $A$-invariant and soluble. We also have $[P, K]=[P, K, K]$ and consequently $[P, K] \leq \gamma_{\infty}(P K)$. Since $H_{1}$ and $H_{2}$ are conjugate to $K$, it follows that both $\left[P, H_{1}\right]$ and $\left[P, H_{2}\right]$ have $\{m, q\}$-bounded rank. 
Let $H=\left\langle H_{1}, H_{2}\right\rangle$. Thus $G=P H$. Since $G=G^{\prime}$ we can deduce that $G=[P, H] H$ because $[P, H] H$ is normal and the quotient $G /[P, H] H$ is a $p$-group. Furthermore, $[P, G]=[P, H]$. Indeed, we have

$$
[P, G]=[P,[P, H] H]=[P,[P, H]][P, H] .
$$

Since $[P, H]$ is normal in $P$ and $G=P H$, the subgroup $[P, H]$ is also normal in $G$ and so $[[P, H], P] \leq[P, H]$.

Note that $[P, H]=\left[P, H_{1}\right]\left[P, H_{2}\right]$. Thus the rank of $[P, H]$ is $\{m, q\}$-bounded. Passing to the quotient $G /[P, G]$ we can assume that $P=Z(G)$. In view of Lemma 4.2 , we know that $G / Z(G)$ has $\{m, q\}$ bounded rank. By a theorem of Lubotzky and Mann [15] (see also [13. Theorem 1.1]) the rank of $G^{\prime}$ is $\{m, q\}$-bounded as well. Since $G=G^{\prime}$ we conclude that the rank of $G$ is $\{m, q\}$-bounded.

Now we deal with the case where $\lambda(G)=1$ and $G \neq G^{\prime}$. Let $G^{(d)}$ be the last term of the derived series of $G$. The argument in the previous paragraph shows that $G^{(d)}$ has $\{m, q\}$-bounded rank. Hence, in virtue of Lemma 4.1. the rank of $\gamma_{\infty}(G)$ is $\{m, q\}$-bounded since $G / G^{(d)}$ is soluble. This proves the theorem in the particular case where $\lambda(G) \leq 1$.

Assume that $\lambda(G) \geq 2$. Let $T$ be a characteristic subgroup of $G$ such that $\lambda(T)=\lambda(G)-1$ and $\lambda(G / T)=1$. By induction, the rank of $\gamma_{\infty}(T)$ is $\{m, q\}$-bounded. Since $\lambda\left(G / \gamma_{\infty}(T)\right)=1$, the rank of the nilpotent residual of $G / \gamma_{\infty}(T)$ is $\{m, q\}$-bounded too. Consequently, we conclude that $\gamma_{\infty}(G)$ has $\{m, q\}$-bounded rank. This completes the proof.

\section{References}

[1] C. Acciarri, P. Shumyatsky, Double automorphisms of graded Lie algebras. J. Algebra, 387 (2013), 1-10.

[2] C. Acciarri, P. Shumyatsky, A. Thillaisundaram, Conciseness of coprime commutators in finite groups, Bull. Aust. Math. Soc.89 (2014), 252-258.

[3] D. Gorenstein, Finite Group, Chelsea Publishing Company, New York, 1980.

[4] R. Guralnick, On the number of generators of a finite group, Arch. Math. 53 (1989), 521-523.

[5] R. Guralnick, P. Shumyatsky, Derived subgroups of fixed points, Israel J. Math. 126, (2001), 345-362.

[6] L. G. Kovács, On finite soluble groups, Math. Z. 103 (1968), 37-39.

[7] E. I. Khukhro, V.D. Mazurov, Finite groups with an automorphism of prime order whose centralizer has small rank, J. Algebra, 301 (2006), 474-492.

[8] E. I. Khukhro, P. Shumyatsky, On the length of finite groups and of fixed points, Proc. Amer. Math. Soc. 143 (2015), 3781-3790.

[9] E. I. Khukhro, P. Shumyatsky, Nonsoluble and non-p-soluble length of finite groups, Israel J. Math. 207 (2015), 507-525. 
[10] E. I. Khukhro, P. Shumyatsky, Almost Engel finite and profinite groups, Internat. J. Algebra Comput. 26 (2016), 973-983.

[11] E. I. Khukhro, P. Shumyatsky, Almost Engel compact groups, J. Algebra, 500 (2018), 439-456.

[12] E. I. Khukhro, P. Shumyatsky, Finite groups with Engel sinks of bounded rank, Glasgow Math. J. (2018), 1-7. doi.org/10.1017/S0017089517000404.

[13] L. A. Kurdachenko, P. Shumyatsky, The ranks of central factor and commutator groups. Math. Proc. Camb. Phil. Soc. 154 (2013), 63-69.

[14] H. Kurzweil, B. Stellmacher, The Theory of Finite Groups: An Introduction, Springer-Verlag, New York, Inc. 2004.

[15] A. Lubotzky, A. Mann, Powerful p-groups I, J. Algebra, 105 (1987), 484-505.

[16] A. Lucchini, A bound on the number of generators of a finite group, Arch. Math. 53 (1989), 313-317.

[17] E. Melo, A.S. Lima, P. Shumyatsky, Nilpotent residual of fixed points, Arch. Math. (2018), 1-9. doi.org/10.1007/s00013-018-1173-1.

[18] D. J.S. Robinson, Finiteness Conditions and Generalized Soluble Groups, Part 1, Springer, Berlin etc., 1972.

[19] D. J. S. Robinson, A Course in the Theory of Groups, 2nd ed. Springer-Verlag, New York, 1996.

[20] J.G. Thompson, Automorphisms of solvable groups, J. Algebra 1 (1964), 259267.

[21] A. Turull, Fitting height of groups and of fixed points, J. Algebra 86 (1984), $555-566$.

[22] M. Zorn, Nilpotency of finite groups, Bull. Amer. Math. Soc. 42 (1936), 485486.

Department of Mathematics, University of Brasilia, 70910-900 Brasília

DF, BRAZIL

E-mail address: acciarricristina@yahoo.it

Department of Mathematics, University of Brasilia, 70910-900 Brasília

DF, BRAZIL

E-mail address: pavel@unb.br

Department of Mathematics, Federal University of Goias, 75704-

020 Catalão GO, Brazil

E-mail address: sancaodanilo@ufg.br 